\title{
Allee effects in a Ricker-type predator-prey system
}

\author{
Yunshyong Chow ${ }^{1}$ and Sophia R.-J. Jang ${ }^{2}$
}

1. Institute of Mathematics, Academia Sinica, Taipei 10617, Taiwan

2. Department of Mathematics and Statistics, Texas Tech University, Lubbock, TX 79409-1042 U.S.A.

\begin{abstract}
We study a discrete host-parasitoid system where the host population follows the classical Ricker functional form and is also subject to Allee effects. We determine basins of attraction of the local attractors of the single population model when the host intrinsic growth rate is not large. In this situation, existence and local stability of the interior steady states for the host-parasitoid interaction are completely analyzed. If the host's intrinsic growth rate is large, then the interaction may support multiple interior steady states. Linear stability of these steady states is provided.
\end{abstract}

AMS Subject Classification. 92D25, 39A30

Key words. Allee effects, Ricker model, period-doubling bifurcation, linear stability

\section{Introduction}

The Allee effect, refers to the reduced fitness or the decline in population growth at low population densities or sizes, was first observed by Allee [1]. It has a significant impact on population survival when the population is at low level. There has been a rebound of interest in Allee effects recently due to fragmentation of habitats, invasions of exotic species, biological control of pest, etc., all involved with small populations. The treatise by Courchamp et al. [5] provides interesting biological motivation and many mathematical models of Allee effects.

The Ricker model [18] is one of the classical equations used to model fish populations and the function has been applied extensively to model other populations as well. Its dynamics are complicated. As the intrinsic growth rate of the population is increased, the map undergoes a cascade of period-doubling bifurcations and eventually becomes chaotic. Due to its importance, the Ricker growth function has been incorporated with Allee effects to study population dynamics by several researchers. Li et al. [14] propose a single population model with Allee effects by assuming a Holling type II functional form for the birth or growth of the population, termed the 
extended Ricker model. The authors [14 compare the dynamics of the extended Ricker model with the classical Ricker model and conclude that Allee effects have the stabilizing effects on population dynamics. In [9], Elaydi and Sacker study a modified Ricker map that exhibits Allee effects. The modified Ricker map is then generalized to include inter-specific interactions of two populations by Livadiotis and Elaydi [17]. Jang and Diamond [11] on the other hand study a predator-prey system with Allee effects occurring in the prey that grows according to the Ricker function. More recently, Kang proposes a two species competition model with Allee effects occurring in both populations [12, where each single population in the absence of the other population follows the growth of the Ricker model and is also subject to Allee effects. There are other mathematical models of Allee effects, including [6, 7, 10, 19, 15, 20, 21] and references cited therein.

In each of these Ricker-type models [9, 11, 12, 14, 17, however, the mechanism of Allee effects is modeled differently from the present single population model studied in this manuscript. Specifically, the way that Allee effects are incorporated into the Ricker equation is the same as that of the deterministic single population model proposed by Allen et al. [2] and by Liebhold and Bascompte [16. To the best of our knowledge, the resulting scalar equation with Allee effects has not been analyzed previously. We derive a threshold $r_{0}$ in terms of the Allee threshold such that the single population possesses only equilibrium dynamics if its intrinsic growth rate $r$ is smaller than the threshold $r_{0}$. Basins of attraction of the local attractors are then explicitly determined. The population exhibits a period-doubling bifurcation at the critical threshold $r=r_{0}$. Based on this single population equation, we build a host-parasitoid system. We study existence and local stability of the interior steady states. Under certain parameter regime, global dynamics are provided for this two-dimensional system. Numerical simulations are also performed using Matlab to study the model.

The host-parasitoid model is presented in Section 2 and the single host population is analyzed in Section 2.1. Section 3 provides analysis of the two-dimensional host-parasiotid system. In particular, preliminary results are given in Section 3.1 and Sections 3.2 and 3.3 deal with the cases of $r<r_{0}$ and $r>r_{0}$ respectively. The final section provides a brief summary.

\section{The model and the host equation}

Let $x(t)$ and $y(t)$ be the host and parasitoid populations at time $t=0,1, \cdots$, respectively. Parameters $r$ and $K$ are the intrinsic growth rate and the carrying capacity of the host population, respectively. Inspired by the studies 
in [4, 13, where the continuous-time logistic model $N^{\prime}(t)=r N(1-N / K)$ is modified to yield $N^{\prime}(t)=r N(1-N / K)(N-a)$ for the mechanism of Allee effects, Liebhold and Bascompte [16] derive the discrete-time version of this type of model for organisms with non-overlapping generations via

$$
\ln (\tilde{\gamma}(t))=r(1-x(t) / K)(x(t)-a),
$$

where $\tilde{\gamma}(t)=x(t+1) / x(t)$ is the change in population density. As a result, one obtains

$$
x(t+1)=x(t) e^{r(1-x(t) / K)(x(t)-a)}
$$

for the single host population that is subject to Allee effect with the Allee threshold given by $a, 0<a<K$. The per capita growth rate of the host population is less than 1 if its population level is below $a$ and this growth rate exceeds 1 if the population level is between $a$ and the carrying capacity $K$. The per capita growth rate is smaller than 1 again if the population level is larger than $K$ due to intra-specific competition.

The parasitoid population feeds on the host population exclusively. Parameter $\beta>0$ is the conversion of prey to predator and $b>0$ represents parasitoid's searching and handling efficiency on the host. The host-parasitoid interaction is given by the following system:

$$
\left\{\begin{array}{l}
x(t+1)=x(t) e^{r(1-x(t) / K)(x(t)-a)} e^{-b y(t)} \\
y(t+1)=\beta x(t)\left(1-e^{-b y(t)}\right)
\end{array}\right.
$$

where $0<a<K$ and $r, b, \beta>0$. There are 5 parameters in model (2.1). We can rescale the state variables and parameters to eliminate some parameters. Let

$$
\hat{x}(t)=x(t) / K, \hat{y}(t)=b y(t), \hat{r}=r K, \hat{a}=a / K, \hat{\beta}=\beta b K .
$$

Then system (2.1) is converted to

$$
\left\{\begin{array}{l}
\hat{x}(t+1)=\hat{x}(t) e^{\hat{r}(1-\hat{x}(t))(\hat{x}(t)-\hat{a})} e^{-\hat{y}(t)} \\
\hat{y}(t+1)=\hat{\beta} \hat{x}(t)\left(1-e^{-\hat{y}(t)}\right),
\end{array}\right.
$$

where $0<\hat{a}<1$. By ignoring the hats, we obtain the following system with only 3 parameters

$$
\left\{\begin{array}{l}
x(t+1)=x(t) e^{r(1-x(t))(x(t)-a)} e^{-y(t)} \\
y(t+1)=\beta x(t)\left(1-e^{-y(t)}\right),
\end{array}\right.
$$

where $r, \beta>0$ and $0<a<1$, and initial conditions are nonnegative. The parameters have different biological meanings than those in model (2.1). We shall study system (2.2) in section 3 and we will focus on the analysis of single host population model in section 2.1. 


\subsection{The host population}

If $y(0)=0$ in (2.2), we have $y(t)=0$ for $t \geq 1$ and (2.2) reduces to a one-dimensional equation

$$
x(t+1)=x(t) e^{r(1-x(t))(x(t)-a)} .
$$

Model (2.3) has three steady states: 0, $a$ and 1. Let $f(x)$ denote the map of $(2.3)$,

$$
f(x)=x e^{r(1-x)(x-a)} .
$$

Then

$$
f^{\prime}(x)=e^{r(1-x)(x-a)}(1+x r(1-2 x+a)),
$$

and

$$
f^{\prime \prime}(x)=r\left(r x(1+a-2 x)^{2}+3(1+a-2 x)-(1+a)\right) e^{r(1-x)(x-a)} .
$$

Since $0<f^{\prime}(0)=e^{-r a}<1$, steady state 0 is always locally asymptotically stable. On the other hand, $f^{\prime}(a)=1+\operatorname{ar}(1-a)>1$, and hence steady state $a$ is a unstable. Since $f^{\prime}(1)=1+r(a-1)<1,1$ is locally asymptotically stable if $r<\frac{2}{1-a}$ and unstable if $r>\frac{2}{1-a}$. We use $r$ as the bifurcation parameter to study (2.3).

Denote $r_{0}$ in terms of the Allee threshold $a$ :

$$
r_{0}=\frac{2}{1-a} .
$$

Then $\left.f^{\prime}(1)\right|_{r=r_{0}}=-1$ and it is expected that a period-doubling bifurcation occurs at $r=r_{0}$. In the following, we first verify that steady state 1 is locally asymptotically stable when $r=r_{0}$. Consider the Schwarzian derivative of $f$,

$$
S f(x)=\frac{f^{\prime \prime \prime}(x)}{f^{\prime}(x)}-\frac{3}{2}\left(\frac{f^{\prime \prime}(x)}{f^{\prime}(x)}\right)^{2} .
$$

By [8, Theorem 1.16], steady state 1 is locally asymptotically stable if $S f(1)<0$ and unstable if $S f(1)>0$. A direct computation yields

$$
\left.f^{\prime \prime}(1)\right|_{r=r_{0}}=-2 r_{0} \text { and }\left.f^{\prime \prime \prime}(1)\right|_{r=r_{0}}=8 r_{0}-2 a r_{0} .
$$

Therefore $\left.S f(1)\right|_{r=r_{0}}=2 r_{0}\left(a-4-3 r_{0}\right)<0$ for $0<a<1$ and steady state 1 is locally asymptotically stable when $r=r_{0}$. To prove period-doubling bifurcation, we apply [8] to verify that

$$
\frac{\partial^{2} f}{\partial x \partial r}\left(1, r_{0}\right) \neq 0 \text { and } \frac{1}{2}\left(\frac{\partial^{2} f}{\partial x^{2}}\left(1, r_{0}\right)\right)^{2}+\frac{1}{3} \frac{\partial^{3} f}{\partial x^{3}}\left(1, r_{0}\right) \neq 0
$$


Notice $\frac{\partial^{2} f}{\partial x \partial r}\left(1, r_{0}\right)=a-1<0$ and the first expression in $(2.9)$ is satisfied. Substituting (2.8) into the second expression of (2.9) arrives at $\frac{2 r_{0}}{3}\left(3 r_{0}+4-\right.$ a) $>0$ and therefore (2.9) holds. As a result, a period-doubling bifurcation occurs at $r=r_{0}$. We now summarize.

Proposition 2.1 Model (2.3) has three steady states 0 , a and 1, where 0 is locally asymptotically stable and $a$ is unstable. Steady state 1 is locally asymptotically stable if $r \leq r_{0}$ and unstable if $r>r_{0}$. Moreover, equation (2.3) undergoes a period-doubling bifurcation at $r=r_{0}$.

It follows from Proposition 2.1 that model (2.3) has 2-cycle solutions when $r>r_{0}$ and is close to $r_{0}$. To further study the existence of 2-cycles, we consider the second composition of $f, f^{2}(x):=f(f(x))$, where

$$
f^{2}(x)=x e^{r(1-x)(x-a)} e^{r\left(1-x e^{r(1-x)(x-a)}\right)\left(x e^{r(1-x)(x-a)}-a\right) .}
$$

Two- cycle solutions are fixed points of the map $f^{2}$ that are not fixed points of $f$. Consequently, components $x$ of any 2-cycles satisfy

$$
(1-x)(x-a)+\left(1-x e^{r(1-x)(x-a)}\right)\left(x e^{r(1-x)(x-a)}-a\right)=0 .
$$

Clearly $a$ and 1 are solutions of (2.11) and we look for other positive solutions. Let $\bar{g}(x)$ denote the left hand side of (2.11). Then $\bar{g}(0)=-2 a<0$, $\bar{g}(a)=\bar{g}(1)=0, \lim _{x \rightarrow \infty} \bar{g}(x)=-\infty$ and

$\bar{g}^{\prime}(x)=a-2 x+1-e^{r(1-x)(x-a)}[1+r x(1-2 x+a)]\left[2 x e^{r(1-x)(x-a)}-a-1\right]$.

Notice $\bar{g}^{\prime}(a)=(1-a)(2+r a(1-a))>0$, and $\bar{g}^{\prime}(1)=(a-1)(2+r(a-1))>0$ if and only if $r>r_{0}$. We conclude that (2.3) has a positive 2-cycle $\mathcal{S}=\left\{\bar{x}_{1}, \bar{x}_{2}\right\}$ if $r>r_{0}$, where $a<\bar{x}_{1}<1<\bar{x}_{2}$.

Notice that $f$ attains its maximum at

$$
x_{m}=\frac{1+a}{4}+\frac{\sqrt{(a+1)^{2} r^{2}+8 r}}{4 r}
$$

where $x_{m}>\frac{1+a}{2}>a$. Moreover, $x_{m}>1$ if and only if $r<r_{0} / 2$, and $x_{m}$ is a decreasing function of $r$. See Figure 1(a)-(b) for the illustration. Notice that there exists a unique $x_{a}>\max \left\{1, x_{m}\right\}$ such that $f\left(x_{a}\right)=a$. Let $\mathcal{B}(x)$ denote the basin of attraction of the steady state $x$. If $x(0)<a$, then $x(1)=$ $f(x(0))<f(a)=a$ and $x(1)=f(x(0))<x(0)$. Inductively, $\{x(t)\}_{t=0}^{\infty}$ is a 
decreasing sequence and bounded below by 0 . Thus, $\lim _{t \rightarrow \infty} x(t)=0$ by the continuity of $f$, i.e., $[0, a) \subset \mathcal{B}(0)$. Furthermore, since $f$ is decreasing on $\left(x_{m}, \infty\right)$, we have $f(x)<a$ if $x>x_{a}$. Therefore, $\lim _{t \rightarrow \infty} x(t)=0$ if $x(0)>x_{a}$ and $[0, a) \cup\left(x_{a}, \infty\right) \subset \mathcal{B}(0)$ for any $r>0$.

In the following, we prove that solutions of (2.3) either converge to $0, a$ or 1 when $r<r_{0}$. We separate the analysis into two cases: $r \leq r_{0} / 2$ and $r_{0} / 2<r<r_{0}$. Notice that $1<x_{m}$ and $1>x_{m}$ when $r<r_{0} / 2$ and $r>r_{0} / 2$, respectively.

Theorem 2.2 If $r \leq r_{0} / 2$, then $\mathcal{B}(0)=[0, a) \cup\left(x_{a}, \infty\right)$ and $\mathcal{B}(1)=\left(a, x_{a}\right)$.

Proof. First assume $r<r_{0} / 2$. Then $x_{m}>1$. If $x \in(a, 1)$, then $f(x)>$ $x>a$ and $f(x)<1$ and hence $\lim _{t \rightarrow \infty} f^{t}(x)=1$. If $x \in\left(1, x_{m}\right]$, then $f(x)<$ $x \leq x_{m}$ and $f(x)>1$ hold and thus $\lim _{t \rightarrow \infty} f^{t}(x)=1$. If $x \in\left(x_{m}, x_{a}\right)$, then $x_{m}>f\left(x_{m}\right)>f(x)>f\left(x_{a}\right)=a$, i.e., $f(x) \in\left(a, x_{m}\right)$, and hence $\lim _{t \rightarrow \infty} f^{t}(x)=1$. Similar analysis can be applied to the case when $r=r_{0} / 2$ and the proof is complete.

Suppose now $r_{0} / 2<r<r_{0}$. Then $x_{m}<1$ and $f^{\prime}(1)<0$. Recall that $\lim _{t \rightarrow \infty} x(t)=0$ if $x(0) \in[0, a) \cup\left(x_{a}, \infty\right)$. We prove that $f\left(x_{m}\right) \geq x_{a}$ cannot occur and the basin of attraction of 1 is $\left(a, x_{a}\right)$.

Theorem 2.3 If $r_{0} / 2<r<r_{0}$, then $f\left(x_{m}\right)<x_{a}$. Moreover, $\mathcal{B}(0)=$ $[0, a) \cup\left(x_{a}, \infty\right)$ and $\mathcal{B}(1)=\left(a, x_{a}\right)$.

Proof. Assume first that $f\left(x_{m}\right)<x_{a}$ holds. Then $\left(a, x_{a}\right)$ is positively invariant for $f, f$ has a maximum at $x_{m} \in(a, 1)$ and $f$ is decreasing on $\left(x_{m}, \infty\right)$. We apply Theorem 2.9 (b) of [3, page 50]. Specifically, since $f$ satisfies (i)-(vi) given in [3, Theorem 2.9(b)], 1 is globally asymptotically stable on $\left(a, x_{a}\right)$ if

$$
f(f(x))>x \text { for } x \in\left[x_{m}, 1\right) .
$$

Using (2.10) and (2.11), (2.14) is equivalent to

$$
(1-x)(x-a)+(1-f(x))(f(x)-a)>0 \text { for } x \in\left[x_{m}, 1\right) .
$$

For $x \in(a, 1)$, let

$$
y=\frac{1+a+\sqrt{(1-a)^{2}+4(1-x)(x-a)}}{2}
$$

be the solution of

$$
(1-x)(x-a)+(1-y)(y-a)=0
$$


that is greater than 1 . Then (2.15) is equivalent to

$$
G(x):=y-f(x)>0 \text { on }\left[x_{m}, 1\right),
$$

where $x_{m}$ depends on $r$ and $a$. As a result, we will need to show that $G(x)>0$ for $x \in\left[x_{m}, 1\right), 0<a<1$ and $r_{0} / 2<r<r_{0}$. Since $f(x)$ is strictly increasing in $r$ for $x \in(a, 1)$, it is enough to show that (2.18) holds at $r=r_{0}$ and $0<a<1$. On the other hand, $G\left(x_{m}\right)>0$ is equivalent $f\left(f\left(x_{m}\right)\right)>x_{m}$ and $f\left(f\left(x_{m}\right)\right)>x_{m}$ implies $f\left(f\left(x_{m}\right)\right)>a$. This later inequality is also equivalent to $f\left(x_{m}\right)<x_{a}$. Therefore, if (2.18) is verified at $r=r_{0}$ and for all $a \in(0,1)$, then $f\left(x_{m}\right)<x_{a}$ holds and the earlier assumption can be dropped. Consequently 1 is globally asymptotically stable on $\left(a, x_{a}\right)$ is also proved. Therefore, we are in a position to verify (2.18) for $r=r_{0}$ and $0<a<1$. If we can show that $G(x)$ is strictly decreasing on $\left[x_{m}, 1\right)$, then since $G(1)=0$ we have $G(x)>0$ on $\left[x_{m}, 1\right)$ and (2.18) is shown. Therefore, in the following we will prove that $G(x)$ is strictly decreasing on $\left[x_{m}, 1\right)$ when $r=r_{0}$ and $0<a<1$.

To this end, differentiating $y$ with respect to $x$, yields

$$
y^{\prime}(x)=\frac{1+a-2 x}{\sqrt{(1-a)^{2}+4(1-x)(x-a)}}<0 \text { on }\left[x_{m}, 1\right)
$$

and

$$
y^{\prime \prime}(x)=\frac{-4(1-a)^{2}}{\left((1-a)^{2}+4(1-x)(x-a)\right)^{3 / 2}}<0 \text { on }\left[x_{m}, 1\right) .
$$

Moreover, it is easy to see from (2.20) that $y^{\prime \prime}(x)$ is strictly decreasing on $\left[x_{m}, 1\right)$. Let

$$
P_{3}(x)=r x(1+a-2 x)^{2}+3(1+a-2 x)-(1+a) .
$$

Then $f^{\prime \prime}(x)=r P_{3}(x) e^{r(1-x)(x-a)}$ by $(2.6)$ and

$$
P_{3}(0)>0, P_{3}\left(\frac{1+a}{2}\right)=-(1+a)<0, \text { and } P_{3}(1)=-2<0 .
$$

Since

$$
\lim _{x \rightarrow-\infty} P_{3}(x)=-\infty \text { and } \lim _{x \rightarrow \infty} P_{3}(x)=\infty,
$$

it follows that $P_{3}(x)<0$ on $\left[x_{m}, 1\right)$ and hence

$$
f^{\prime \prime}(x)<0 \text { on }\left[x_{m}, 1\right) .
$$


A straightforward computation shows that

$$
f^{\prime \prime \prime}(x)=r P_{4}(x) e^{r(1-x)(x-a)}
$$

where

$$
P_{4}(x)=-\frac{r^{2}}{2} z^{4}-\frac{r^{2}(1+a)}{2} z^{3}+6 r z^{2}+3 r(1+a) z-6
$$

and $z=2 x-1-a$. If we can show that $P_{4}(x)>0$ on $\left[x_{m}, 1\right)$, then $f^{\prime \prime \prime}(x)>0$ on $\left[x_{m}, 1\right)$, which implies $f^{\prime \prime}(x)$ is strictly increasing on $\left[x_{m}, 1\right)$. Since $y^{\prime \prime}(x)$ is strictly decreasing on $\left[x_{m}, 1\right)$ by $(2.20)$, we see that $G^{\prime \prime}(x)=y^{\prime \prime}(x)-f^{\prime \prime}(x)$ is strictly decreasing on $\left[x_{m}, 1\right)$. Now, $y^{\prime \prime}(1)=-\frac{4}{1-a}$ and $f^{\prime \prime}(1)=-\frac{4}{1-a}$ at $r=r_{0}$ imply $G^{\prime \prime}(1)=0$. Hence $G^{\prime \prime}(x)>0$ on $\left[x_{m}, 1\right)$ and $G^{\prime}(x)$ is strictly increasing on $\left[x_{m}, 1\right)$. Since $G^{\prime}(1)=y^{\prime}(1)-f^{\prime}(1)=0$, we must have $G^{\prime}(x)<0$ on $\left[x_{m}, 1\right)$. As $G(1)=y(1)-f(1)=0$, we conclude that $G(x)>0$ on $\left[x_{m}, 1\right)$.

It remain to prove that $P_{4}(x)>0$ on $\left[x_{m}, 1\right)$. Differentiating $P_{4}(x)$ yields $P_{4}^{\prime}(x)=-4 r^{2}(2 x-1-a)^{2}-3 r^{2}(1+a)(2 x-1-a)^{2}+24 r(2 x-1-a)+6 r(1+a)$

and

$$
P_{4}^{\prime \prime}(x)=-12 r\left(2 r(2 x-1-a)^{2}+r(1+a)(2 x-1-a)-4\right) .
$$

Solving $P_{4}^{\prime \prime}(x)=0$, we obtain

$$
x_{ \pm}=\frac{1+a}{2}-\frac{1+a}{8} \pm \frac{\sqrt{r^{2}(1+a)^{2}+32 r}}{8 r} .
$$

Notice

$$
x_{-}<\frac{1+a}{2}<x_{+}, \text {and } x_{+}<1 \text { if and only if } 8(a-1)^{2}>0 .
$$

This later inequality holds trivially since $a<1$ and hence $x_{+}<1$ is valid. Furthermore, the graph of $y=P_{4}^{\prime \prime}(x)$ is concave down, and $P_{4}^{\prime \prime}(x)$ is positive on $\left(x_{-}, x_{+}\right)$and negative on $\left(x_{+}, \infty\right) \cup\left(-\infty, x_{-}\right)$. As a result, the graph of $y=P_{4}^{\prime}(x)$ has critical points at $x=x_{ \pm}$and $P_{4}^{\prime}$ is increasing on $\left(x_{-}, x_{+}\right)$ and decreasing on $\left(x_{+}, \infty\right) \cup\left(-\infty, x_{-}\right)$. Since

$$
P_{4}^{\prime}\left(\frac{1+a}{2}\right)=6 r(1+a)>0
$$


and

$$
P_{4}^{\prime}(1)=-16(1-a)-12(1+a)+48+\frac{12}{1-a}(1+a)>0
$$

we conclude that $P_{4}^{\prime}(x)>0$ on $\left[\frac{1+a}{2}, 1\right]$. We need to determine the sign of $P_{4}\left(x_{m}\right)$. Since $x_{m}$ satisfies $1+r x(1+a-2 x)=0$, which implies $2 x r(2 x-$ $1-a)-2=0$ and hence $x_{m}$ solves

$$
r(2 x-1-a)^{2}+r(1+a)(2 x-1-a)-2=0 .
$$

Using (2.25) and long division, we obtain

$$
P_{4}\left(x_{m}\right)=-2 r(1+a)\left(2 x_{m}-1-a\right)+4 .
$$

It follows that $P_{4}\left(x_{m}\right)>0$ at $r=r_{0}$. Consequently, $P_{4}(x)>0$ on $\left[x_{m}, 1\right)$ and the proof is now complete.

Recall that steady state 0 is always locally asymptotically stable and steady state 1 is locally asymptotically stable if $r<r_{0}$. Theorems 2.2 and 2.3 provide basins of attractions for these two attractors when $r<r_{0}$. Specifically, $\mathcal{B}(0)=[0, a) \cup\left(x_{a}, \infty\right)$ and $\mathcal{B}(1)=\left(a, x_{a}\right)$ whenever $r<r_{0}$. Moreover, (2.3) has a period two solution $\mathcal{S}=\left\{\bar{x}_{1}, \bar{x}_{2}\right\}$ when $r>r_{0}$, where $a<\bar{x}_{1}<1<\bar{x}_{2}$. The local stability of $\mathcal{S}$ can be determined by the derivative of $f^{2}(x)$ evaluated at either $\bar{x}_{1}$ or $\bar{x}_{2}$. Since $f\left(\bar{x}_{1}\right)=\bar{x}_{2}$, a simple calculation shows

$$
\begin{aligned}
& \left.\left(f^{2}(x)\right)^{\prime}\right|_{x=\bar{x}_{1}}=f^{\prime}\left(\bar{x}_{1}\right) f^{\prime}\left(\bar{x}_{2}\right) \\
& =e^{r\left(\left(1-\bar{x}_{1}\right)\left(\bar{x}_{1}-a\right)+\left(1-\bar{x}_{2}\right)\left(\bar{x}_{2}-a\right)\right)}\left[1+r \bar{x}_{1}\left(1-2 \bar{x}_{1}+a\right)\right]\left[1+r \bar{x}_{2}\left(1-2 \bar{x}_{2}+a\right)\right] .
\end{aligned}
$$

Using (2.11) and $a<\bar{x}_{1}<1$, we have $\bar{x}_{1} e^{r\left(1-\bar{x}_{1}\right)\left(\bar{x}_{1}-a\right)}<1$ and hence $\bar{x}_{1}<x_{m}$. It follows that

$$
1+r \bar{x}_{1}\left(1-2 \bar{x}_{1}+a\right)>0
$$

and

$1+r \bar{x}_{2}\left(1-2 \bar{x}_{2}+a\right)<1+r \bar{x}_{2}(a-1)<1-r(1-a)<1-r_{0}(1-a)=-1<0$.

As a result,

$$
\left.\left(f^{2}(x)\right)^{\prime}\right|_{x=\bar{x}_{1}}<0 .
$$

Therefore, $\mathcal{S}$ is locally asymptotically stable if $\left.\left(f^{2}(x)\right)^{\prime}\right|_{x=\bar{x}_{1}}>-1$ and unstable if $\left.\left(f^{2}(x)\right)^{\prime}\right|_{x=\bar{x}_{1}}<-1$. Numerically, we can find the 2-cycle solution using 
(2.11) and then determine its local stability using (2.27) when $r>r_{0}$. It is expected that (2.3) undergoes another period-doubling bifurcation when $\left.\left(f^{2}(x)\right)^{\prime}\right|_{x=\bar{x}_{1}}=-1$. Figure $1(\mathrm{c})$ provides a bifurcation diagram for (2.3) when $a=0.5$ and using $r$ as the bifurcation parameter. The figure conforms with our analytical findings.

\section{The host-parasitoid model}

In this section, we study the full system (2.2). Preliminary results are provided in Section 3.1. Stability and existence of interior steady states when $r<r_{0}$ and $\beta>1$ are given in Section 3.2. Section 3.3 treats the case for $r>r_{0}$ and $\beta>1$.

\subsection{Preliminary properties of the host-parasitoid system}

Let $F(x, y)$ denote the map induced by $(2.2)$, i.e.,

$$
F(x, y)=\left(f_{1}(x, y), f_{2}(x, y)\right)
$$

where

$$
f_{1}(x, y)=x e^{r(1-x)(x-a)-y} \text { and } f_{2}(x, y)=\beta x\left(1-e^{-y}\right) .
$$

Notice $f_{1}(x, y)=f(x) e^{-y}, x_{a}>\max \left\{x_{m}, 1\right\}$ is the unique $x$ value for which $f\left(x_{a}\right)=a$, and $x_{m}$ is the point for which $f$ attains its maximum. Define the following two regions:

$$
\begin{aligned}
& \Gamma=\left\{(x, y) \in \mathbb{R}_{+}^{2}: x \leq a \text { and }(x, y) \neq(a, 0)\right\} \\
& \Delta=\left\{(x, y) \in \mathbb{R}_{+}^{2}: x \geq x_{a} \text { and }(x, y) \neq\left(x_{a}, 0\right)\right\} .
\end{aligned}
$$

We prove that both populations go extinct if the initial host population is either too small or too large as given in Proposition 3.1.

Proposition 3.1 Solutions of (2.2) remain nonnegative and are bounded for $t>0$. Moreover, solutions $(x(t), y(t))$ of (2.2) with $(x(0), y(0)) \in \Gamma \cup \Delta$ converge to $E_{0}=(0,0)$.

Proof. Let $(x(t), y(t))$ be an arbitrary solution of $(2.2)$. It is clear that the solution exists and remains nonnegative for $t>0$. Moreover, $x(t+$ $1)=f_{1}(x(t), y(t)) \leq f(x(t))$ for $t \geq 0$. Since $f$ attains its maximum at $x=x_{m}, x(t+1) \leq f\left(x_{m}\right)$ and thus $y(t+1) \leq \beta x(t) \leq \beta f\left(x_{m}\right)$ for $t>0$. Therefore, solutions are bounded for $t>0$. If $x(0) \geq x_{a}$ and $y(0)>0$, then $x(1)=f_{1}(x(0), y(0))<f(x(0)) \leq f\left(x_{a}\right)=a$ and hence such a solution 
lies in $\Gamma$ for $t \geq 1$. Suppose now $x(0) \leq a$ and $y(0)>0$. Then $x(1)=$ $f_{1}(x(0), y(0))<f(x(0)) \leq x(0) \leq a$ and hence $\lim _{t \rightarrow \infty} x(t)=0$. As a result, $\lim _{t \rightarrow \infty} y(t)=0$ and the solution converges to $E_{0}$.

Let $a<x(0)<1$. Setting $x(1)=x(0) e^{r(1-x(0))(x(0)-a)} e^{-y(0)} \leq a$ and solving for $y(0)$, yield

$$
y(0) \geq \ln \left(\frac{x(0)}{a}\right)+(1-x(0))(x(0)-a) .
$$

That is, if $a<x(0)<1$ and $y(0)$ satisfies (3.3), then $x(1) \leq a$ and the solution converges to $E_{0}$ by Proposition 3.1. We summarize below.

Proposition 3.2 If $a<x(0)<1$ and $y(0)$ satisfies (3.3), then the solution converges to $E_{0}=(0,0)$.

System (2.2) has two more boundary steady states: $E_{1}=(a, 0)$ and $E_{2}=(1,0)$. The Jacobian matrix of $(2.2)$ evaluated at the boundary steady states are given respectively by

$$
\begin{gathered}
J\left(E_{0}\right)=\left(\begin{array}{cc}
e^{-a r} & 0 \\
0 & 0
\end{array}\right), J\left(E_{1}\right)=\left(\begin{array}{cc}
1+a r(1-a) & -a \\
0 & \beta a
\end{array}\right), \\
J\left(E_{2}\right)=\left(\begin{array}{cc}
1-r(1-a) & -1 \\
0 & \beta
\end{array}\right) .
\end{gathered}
$$

Since each of the above Jacobian matrices is triangular, local stability of these steady states can be easily determined and are summarized below.

Proposition 3.3 System (2.2) has three boundary steady states $E_{0}=(0,0)$, $E_{1}=(a, 0)$ and $E_{2}=(1,0)$, where $E_{0}$ is locally asymptotically stable, $E_{1}$ is a saddle point if $\beta a<1$ and a repeller if $\beta a>1$. Steady state $E_{2}$ is locally asymptotically stable if $r<r_{0}$ and $\beta<1$ and unstable if either $r>r_{0}$ or $\beta>1$.

An interior steady state $(x, y)$ of $(2.2)$ satisfies

$$
y=g(x):=r(1-x)(x-a)
$$

and

$$
x=h(y):=\frac{y}{\beta\left(1-e^{-y}\right)} .
$$

It is clear that $g(x)$ is a concave down parabola with vertex at

$$
\hat{x}=\frac{1+a}{2}
$$


and goes through the points $(a, 0)$ and $(1,0)$. On the other hand,

$$
h^{\prime}(y)=\frac{e^{-y}\left(e^{y}-1-y\right)}{\beta\left(1-e^{-y}\right)^{2}}>0 \text { for } y>0
$$

and

$$
h^{\prime \prime}(y)=\frac{e^{-2 y}\left((y-2) e^{y}+y+2\right)}{\beta\left(1-e^{-y}\right)^{3}}>0 \text { for } y>0 .
$$

Hence $h(y)$ is increasing and concave up on $(0, \infty)$ with $\lim _{y \rightarrow 0^{+}} h(y)=1 / \beta$ and $\lim _{y \rightarrow \infty} h(y)=\infty$. Consequently, if $\beta \leq 1$, then the two curves $y=g(x)$ and $x=h(y)$ have no positive intersections and (2.2) has no interior steady state. In such a case, $\beta a<\beta \leq 1$ and $E_{1}=(a, 0)$ is a saddle point with a one-dimensional local stable manifold $\gamma$ by Proposition 3.3. In the following, we estimate $\gamma$.

Notice that an eigenvector of $J\left(E_{1}\right)$ belonging to the eigenvalue $\beta a$ can be chosen to be $V=\left(\frac{a}{1-\beta a+a r(1-r)}, 1\right)^{T}$. Letting $u=x-a$ and $v=y$, system $(2.2)$ is transformed to

$$
\left\{\begin{array}{l}
u(t+1)=(u(t)+a) e^{r u(t)(1-u(t)-a)} e^{-v(t)}-a \\
v(t+1)=\beta(u(t)+a)\left(1-e^{-v(t)}\right) .
\end{array}\right.
$$

Steady state $E_{1}=(a, 0)$ of $(2.2)$ becomes steady state $(0,0)$ of (3.8). Let $v=\gamma(u)=c_{1} u+c_{2} u^{2}+O\left(u^{3}\right)$ for $u$ near 0 be the local stable manifold of $(0,0)$. Since eigenvector $V$ is tangent to $\gamma$ at $(0,0), c_{1}$ is the slope of $V$, i.e., $c_{1}=\frac{1-\beta a+a r(1-r)}{a}$. Using the positive invariance of $\gamma$ with respect to system (3.8), we have

$$
\begin{aligned}
& v(t+1)=\gamma\left((u(t)+a) e^{r u(t)(1-u(t)-a)-v(t)}-a\right) \\
& =\beta(u(t)+a)\left[1-e^{-c_{1} u(t)-c_{2} u^{2}(t)+O\left(u^{3}(t)\right)}\right] .
\end{aligned}
$$

Equating the expressions with respect to $u$, we obtain $c_{2}=\frac{1+\operatorname{ar}(1-a)}{a^{2}}$, i.e.,

$$
\gamma(u)=\frac{1-\beta a+a r(1-a)}{a} u+\frac{1+a r(1-a)}{a^{2}} u^{2}+O\left(u^{3}\right) \text { for } u \text { near } 0 .
$$

Converting back to the original state variables $x$ and $y$, yields

$$
\gamma(x)=\beta a-\frac{\beta a+1+a r(1-a)}{a} x+\frac{1+a r(1-a)}{a^{2}} x^{2}+O\left(x^{3}\right)
$$


for $x$ near $a$.

Proposition 3.4 Let $\beta \leq 1$. Then system (2.2) has no interior steady state and $E_{1}$ is a saddle point with the local stable manifold given by (3.9). If $\beta<1$ and $r<r_{0}$, then $\lim _{t \rightarrow \infty} y(t)=0$ and $\lim _{t \rightarrow \infty}(x(t), y(t))=E_{0}, E_{1}$, or $E_{2}$ for all solutions $(x(t), y(t))$ of (2.2).

Proof. We only need to prove the last statement for $y(0)>0$. Since $r<r_{0}$, solutions of $(2.3)$ satisfy $\lim \sup x(t) \leq 1$ by Theorems 2.2 and 2.3. Hence for any $\epsilon>0$ there exists $t_{0}>0$ such that $x(t)<1+\epsilon$ for all $t \geq t_{0}$ and for all solutions of (2.3). We can choose $\epsilon>0$ so that $\beta(1+\epsilon)<1$ by the assumption $\beta<1$. Then $y(t+1)<\beta(1+\epsilon)\left(1-e^{-y(t)}\right)$ for all $t \geq t_{0}$. Hence $\lim _{t \rightarrow \infty} y(t)=0$ and system (2.2) is asymptotic to the scalar equation (2.3). Therefore, solutions of (2.2) either converge to $E_{0}, E_{1}$, or $E_{2}$ by Theorems 2.2 and 2.3 .

\subsection{Existence and local stability of the interior steady states when $r<r_{0}$ and $\beta>1$}

If $r<r_{0}$, then the asymptotic dynamics of the host population is completely determined. Moreover, (2.2) has no interior steady state if $\beta \leq 1$ by Proposition 3.4. Let $\beta>1$. We show in this subsection that the host-parasitoid system (2.2) has at most one interior steady state and local stability of the unique interior steady state is presented. We first prove that (2.2) has no interior steady state if $0<\frac{1}{\beta} \leq a$ and (2.2) has a unique interior steady state if $a<\frac{1}{\beta}<1$.

Theorem 3.5 Let $r<r_{0}$ and $\beta>1$. System (2.2) has no interior steady state if $0<\frac{1}{\beta} \leq a$ and (2.2) has a unique interior steady state if $a<\frac{1}{\beta}<1$.

Proof. From previous analysis on the isoclines, (2.2) has at least one interior steady state when $a<\frac{1}{\beta}<1$. If there exists $\beta>1$ such that that the two isoclines have two intersections $\left(x_{i}, y_{i}\right), i=1,2$, such that $a<x_{1}<x_{2}<1$, then $\frac{1}{\beta}$ as a function of $x$

$$
\frac{1}{\beta}(x)=\frac{x\left(1-e^{-y}\right)}{y},
$$


where $y=r(1-x)(x-a)$, has a critical point in $\left(x_{1}, x_{2}\right)$. However, a straightforward calculation shows that $\left(\frac{1}{\beta}\right)^{\prime}(x)>0$ for all $x \in(a, 1)$ if $r \leq r_{0}$. Indeed,

$$
\left(\frac{1}{\beta}\right)^{\prime}=\frac{\left(1-e^{-y}\right)}{y}+x \frac{(1+y) e^{-y}-1}{y^{2}} \cdot r(1+a-2 x),
$$

where $(1+y) e^{-y}-1<0$ for $y>0$ and $r x(1+a-2 x) \leq r(1-a) \leq 2$ for $x \in(a, 1)$ since $r \leq r_{0}$. Hence

$$
\left(\frac{1}{\beta}\right)^{\prime} \geq \frac{y-2+(y+2) e^{-y}}{y^{2}},
$$

where

$$
\left(y-2+(y+2) e^{-y}\right)^{\prime}=1-(1+y) e^{-y}>0 \text { for } y>0
$$

and

$$
\left.\left(y-2+(y+2) e^{-y}\right)\right|_{y=0}=0 .
$$

Thus $\left(\frac{1}{\beta}\right)^{\prime}(x)>0$ for all $x \in(a, 1)$ and $(2.2)$ has a unique interior steady state if $a<\frac{1}{\beta}<1$. When $\beta a=1$, then since $(a, 0)$ is itself a steady state, (2.2) has no interior steady state. Moreover, since $\frac{y}{1-e^{-y}}=\beta x$ is an increasing function of $\beta$ if $x$ is kept fixed, (2.2) has no interior steady state if $\beta a>1$.

The Jacobian matrix of (2.2) evaluated at any interior steady state $(x, y)$ can be easily shown to be

$$
J=\left(\begin{array}{cc}
1+x r(1-2 x+a) & -x \\
\frac{y}{x} & \beta x e^{-y}
\end{array}\right),
$$

with

$$
\operatorname{tr} J=1+x r(1-2 x+a)+\beta x e^{-y}
$$

and

$$
\operatorname{det} J=\beta x e^{-y}(1+x r(1-2 x+a))+y,
$$

where $y=g(x)$. Using the second equilibrium equation, we have $\beta=$ $\frac{y}{x\left(1-e^{-y}\right)}=\frac{y e^{y}}{x\left(e^{y}-1\right)}<\frac{e^{y}}{x}$. Thus for any interior steady state $(x, y)$ there holds

$$
\beta x e^{-y}<1
$$


Moreover, $\max \left\{\frac{1}{\beta}, a\right\}<x<1$ is always satisfied.

It is well known that $(x, y)$ is locally asymptotically stable if $|\operatorname{tr} J|<$ $1+\operatorname{det} J$ and $\operatorname{det} J<1[3]$. Notice $\operatorname{tr} J>-1-\operatorname{det} J$ is equivalent to

$$
2+x r(1-2 x+a)+\beta x e^{-y}(2+x r(1-2 x+a))+y>0 .
$$

Since $r<r_{0}$, we have $f^{\prime}(x)>f^{\prime}(1)>-1$ if $x \geq x_{m}$ by (2.22) and hence

$$
1+e^{r(1-x)(x-a)}(1+x r(1-2 x+a))>0 .
$$

If $x<x_{m}$, then $1+x r(1-2 x+a)>0$ and the above inequality holds trivially. Therefore, $2+x r(1-2 x+a)>1-e^{-r(1-x)(x-a)}>0$ and $\operatorname{tr} J>-1-\operatorname{det} J$ is valid for any interior steady state of (2.2) whenever $r<r_{0}$. On the other hand, $\operatorname{tr} J<1+\operatorname{det} J$ is equivalent to

$$
\operatorname{xr}(1-2 x+a)\left(1-\beta x e^{-y}\right)<y .
$$

This last inequality is satisfied by (3.14) if $x \geq \hat{x}$.

The determinant of $J$ given in (3.13) can be rewritten as a function of $x$ :

$$
D(x)=(1+r x(1+a-2 x)) \cdot \frac{y e^{-y}}{1-e^{-y}}+y,
$$

where $y=r(1-x)(x-a)$. Since

$$
\begin{gathered}
D(\hat{x})=1 \cdot \frac{\hat{y} e^{-\hat{y}}}{1-e^{-\hat{y}}}+\hat{y}=\frac{\hat{y}}{1-e^{-\hat{y}}}>1, \\
\left.D(x)\right|_{x \geq x_{m}} \leq 0+y=r(1-x)(x-a) \leq r\left(\frac{1-a}{2}\right)^{2}<0.5 \text { as } r<r_{0},
\end{gathered}
$$

$D(x)=1$ has at least one solution on $\left[\hat{x}, x_{m}\right)$. We prove that $D(x)$ is strictly decreasing on $[\hat{x}, 1)$. By a direct differentiation, we have

$$
\begin{aligned}
& D^{\prime}(x)=r(1+a-4 x) \cdot \frac{y e^{-y}}{1-e^{-y}}+r x \cdot \frac{d}{d y}\left(\frac{y e^{-y}}{1-e^{-y}}\right) \cdot y^{\prime}+\frac{1-e^{-y}-y e^{-y}}{\left(1-e^{-y}\right)^{2}} \cdot y^{\prime} \\
& =r(1+a-4 x) \cdot \frac{y e^{-y}}{1-e^{-y}}+r^{2} x(1+a-2 x)^{2} \cdot \frac{(1-y) e^{y}-1}{\left(1-e^{-y}\right)^{2}}+\frac{1-(1+y) e^{-y}}{\left(1-e^{-y}\right)^{2}} \cdot r(1+a-2 x) .
\end{aligned}
$$

Since

$$
1-y<e^{-y} \text { and } e^{y}>1+y \text { for all } y>0,
$$

$D^{\prime}(x)<0$ on $[\hat{x}, 1)$. 
If $r \in\left[r_{0} / 2, r_{0}\right)$, then $x_{m} \leq 1$ and $D(x)=1$ has a unique positive solution $\underline{x}$ on $\left(\hat{x}, x_{m}\right)$ with

$$
D(x)>1 \text { on }[\hat{x}, \underline{x}) \text { and } D(x)<1 \text { on }(\underline{x}, 1) .
$$

If $r \in\left(0, r_{0} / 2\right)$, then $1<x_{m}$ and we have either $D(x)>1$ on $[\hat{x}, 1)$ when $\underline{x} \in\left(1, x_{m}\right)$ or there exists a unique $\underline{x} \in(\hat{x}, 1) \subset\left(\hat{x}, x_{m}\right)$ such that (3.16) holds.

Theorem 3.6 Let $r<r_{0}$ and $a<\frac{1}{\beta}<1$ and let $E=(x, y)$ be the unique interior steady state of (2.2). Then $E$ is locally asymptotically stable if $x>\underline{x}$ and $E$ is a repeller if $x<\underline{x}$.

Proof. Assume first $x>\underline{x}$. We can exclude the discussion for $\underline{x} \in\left(1, x_{m}\right)$ since $(x, y)$ is not an interior steady state for $x>\underline{x}>1$. Then $\operatorname{det} J<1$ by (3.16) and $\operatorname{tr} J>-1-\operatorname{det} J$ is satisfied since $r<r_{0}$ by the previous discussion. Furthermore, $\operatorname{tr} J<1+\operatorname{det} J$ is also valid as $x>\underline{x}>\hat{x}$. Therefore $|\operatorname{tr} J|<1+\operatorname{det} J$ is proven and $E$ is locally asymptotically stable if $x>\underline{x}$.

Suppose now $x<\underline{x}$. We separate the discussion into $x \leq \hat{x}$ and $x>\hat{x}$. If $x \leq \hat{x}$, then since $x>\frac{1}{\beta}$, (3.13) implies

$$
\operatorname{det} J=\beta x+r \beta x^{2} e^{-y}(1-2 x+a) \geq \beta x>1 .
$$

To prove $E$ is a repeller, it is equivalent to verify that both of the eigenvalues $\lambda_{ \pm}$of $J$ have modulus greater than 1 . If $(\operatorname{tr} J)^{2}-4 \operatorname{det} J<0$, then the eigenvalues are complex with modulus greater than 1 by the fact that $\left|\lambda_{ \pm}\right|^{2}=$ $\left|\lambda_{+} \lambda_{-}\right|=\operatorname{det} J>1$. If $(\operatorname{tr} J)^{2}-4 \operatorname{det} J \geq 0$, then both $\lambda_{ \pm}$are real and $(\operatorname{tr} J)^{2} \geq 4 \operatorname{det} J>4$. In this case, $\min \left|\lambda_{ \pm}\right|>1$ if and only if $|\operatorname{tr} J|<1+\operatorname{det} J$. Since $x \leq \hat{x}<x_{m}$, we have $\operatorname{tr} J>0$. Therefore, $|\operatorname{tr} J|=\operatorname{tr} J<1+\operatorname{det} J$ is equivalent to

$$
r\left(x(1+a-2 x)-(1-x)(x-a)-x(1+a-2 x) \cdot \frac{y}{e^{y}-1}\right)<0,
$$

where $y=r(1-x)(x-a)$.

Let the left hand side of the above inequality be denoted by $V(x), a<$ $x \leq \hat{x}$. Then $\lim _{x \rightarrow a^{+}} V(x)=0$ since $\lim _{y \rightarrow 0^{+}} \frac{y}{e^{y}-1}=1$. If we can show that $V^{\prime}(x)<0$ on $(a, \hat{x})$, then $V(x)<0$ on $(a, \hat{x})$ is proved and $E$ is a repeller. A straightforward differentiation yields

$$
V^{\prime}(x)=-r\left((1+a-2 x) \frac{y}{e^{y}-1}\left(1-\frac{x r(1-2 x+a)}{2}\right)+B\right),
$$


where

$$
B=x\left(2\left(1-\frac{y}{e^{y}-1}\right)+r(1-2 x+a)^{2} \cdot\left(\frac{(2-y) e^{y}-(2+y)}{2\left(e^{y}-1\right)^{2}}\right)\right) .
$$

Since

$$
\left((2-y) e^{y}-(2+y)\right)^{\prime}=e^{y}\left((1-y)-e^{-y}\right)<0 \text { for } y>0
$$

and

$$
\left.\left((2-y) e^{y}-(2+y)\right)\right|_{y=0}=0,
$$

we have $(2-y) e^{y}-(2+y)<0$ for $y>0$. Also, $r(1-2 x+a)^{2}<r_{0}(1-a)^{2}<2$ on $(a, \hat{x}]$. Hence for $a<x \leq \hat{x}$,

$B \geq 2 x\left(\left(1-\frac{y}{e^{y}-1}\right)+\frac{(2-y) e^{y}-(2+y)}{2\left(e^{y}-1\right)^{2}}\right)=2 x\left(\frac{2 e^{2 y}-(2+3 y) e^{y}+y}{2\left(e^{y}-1\right)^{2}}\right)$.

Since

$$
\begin{gathered}
\left(2 e^{2 y}-(2+3 y) e^{y}+y\right)^{\prime}=4 e^{2 y}-(5+3 y) e^{y}+1 \\
\geq(4(1+y)-(5+3 y)) e^{y}+1>e^{y}\left(e^{-y}-(1-y)\right)>0
\end{gathered}
$$

for $y>0$ and $\left.\left(2 e^{2 y}-(2+3 y) e^{y}+y\right)\right|_{y=0}=0$, we have $2 e^{2 y}-(2+3 y) e^{y}+y>0$ for $y>0$, i.e., for $x \in(a, \hat{x}]$. This shows that $B>0$ on $(a, \hat{x}]$. Moreover, $1+a-2 x<1-a$ and hence $\frac{x r(1-2 x+a)}{2}<\frac{r(1-a)}{2}<1$ for all $x \in(a, \hat{x}]$. Therefore, $V^{\prime}(x)<0$ on $(a, \hat{x}]$ and $V(x)<0$ on $(a, \hat{x}]$ is verified. It follows that the interior steady state $E$ is a repeller if $x \leq \hat{x}$. The proof of $x \in(\hat{x}, \underline{x})$ is trivial since $\operatorname{det} J>1$ by (3.16) and $\operatorname{tr} J>-1-\operatorname{det} J$ always holds. Moreover, $\operatorname{tr} J<1+\operatorname{det} J$ from an earlier observation since $x>\hat{x}$. Therefore, $|\operatorname{tr} J|<1+\operatorname{det} J$ and $E$ is a repeller if $x \in(\hat{x}, \underline{x})$.

In particular, the interior steady state $E=(x, y)$ is locally asymptotically stable when $x$ is near 1 . Finally, we prove that steady state $E_{0}=(0,0)$ is globally asymptotically stable in $\left\{(x, y) \in \mathbb{R}_{+}^{2}: y>0\right\}$ if $\beta a>e^{\frac{1-a}{2}}$ and $r<r_{0}$.

Theorem 3.7 If $\beta a>e^{\frac{1-a}{2}}$ and $r<r_{0}$, then $E_{0}=(0,0)$ is globally asymptotically stable in $\left\{(x, y) \in \mathbb{R}_{+}^{2}: y>0\right\}$ for system (2.2).

Proof. Let $(x(t), y(t))$ be an arbitrary solution of (2.2) with $x(0), y(0)>0$. Then $x(t), y(t)>0$ for $t \geq 0$. If there exists $t_{0}>0$ such that $x\left(t_{0}\right) \leq a$, then $\lim _{t \rightarrow \infty}(x(t), y(t))=E_{0}$ by Proposition 3.1. Assume now $x(t)>a$ for all $t \geq 0$. Since $r(1-x)(x-a)<r_{0}(1-\hat{x})(\hat{x}-a)<\frac{1-a}{2}$ for all $x \geq a$, we 
have $x(t+1) \leq x(t) e^{\frac{1-a}{2}} e^{-y(t)}$ and $y(t+1) \geq \beta a\left(1-e^{-y(t)}\right)$ for all $t>0$. Consider

$$
z(t+1)=\beta a\left(1-e^{-z(t)}\right) .
$$

The scalar equation (3.18) has a unique interior steady state $\bar{z}$ and $z(t) \rightarrow \bar{z}$ as $t \rightarrow \infty$ if $z(0)>0$. It follows that $\liminf _{t \rightarrow \infty} y(t) \geq \bar{z}$.

We verify that $\bar{z}>\frac{1-a}{2}$. Let $S(z)=\beta a\left(1-e^{-z}\right)-z, z \geq 0$. Then $S(0)=0$ and $S^{\prime}(z)=\beta a e^{-z}-1>0$ for $0 \leq z \leq \frac{1-a}{2}$ by the assumption, i.e., $S(z)>0$ for $0<z \leq \frac{1-a}{2}$. Hence $\beta a\left(1-e^{-z}\right)>z$ for $0<z \leq \frac{1-a}{2}$ and $\bar{z}>\frac{1-a}{2}$ is shown. For any $\epsilon>0$ there exists $t_{1}>0$ such that $y(t)>\bar{z}-\epsilon$ for all $t \geq t_{1}$. We can choose $\epsilon>0$ such that $\frac{1-a}{2}+\epsilon-\bar{z}<0$. Then

$$
x(t+1) \leq x(t) e^{\frac{1-a}{2}+\epsilon-\bar{z}} \text { for } t \geq t_{1}
$$

implies $\lim _{t \rightarrow \infty} x(t)=0$. Therefore, the solution converges to $E_{0}$ and $E_{0}$ is globally asymptotically stable in $\left\{(x, y) \in \mathbb{R}_{+}^{2}: y>0\right\}$ since the solution was arbitrary and $E_{0}$ is locally asymptotically stable.

Dynamical behavior of system (2.2) is now summarized for $r<r_{0}$ and is illustrated in Figure 2. We do not analytically prove local bifurcations of (2.2).

- $\beta<1$ : (2.2) has no interior steady state and solutions converge to one of the boundary steady states $E_{0}, E_{1}$ or $E_{2}$ depending on initial conditions. It is expected that the system undergoes a transcritical bifurcation at $\beta=1$ and (2.2) has an asymptotically stable interior steady state when $\beta>1$ and is near 1 .

- $1<\beta<\frac{1}{a}$ : $(2.2)$ has a unique interior steady state $E=(x, y)$, where $E$ is locally asymptotically stable if $x>\underline{x}$ and is a repeller if $x<\underline{x}$. As $|\operatorname{tr} J|<1+\operatorname{det} J$ holds for any interior steady state $E=(x, y)$ while $D(\underline{x})=1$ and $D^{\prime}(x)<0$ on $[\hat{x}, 1)$, one expects that a Neimark-Sacker bifurcation occurs when $x=\underline{x}$. Recall that $\frac{1}{\beta}$ is a strictly increasing function of $x$. Therefore, there exists a unique $\beta_{c}, 1<\beta_{c}<\frac{1}{a}$, that corresponds to the unique $\underline{x}$. Moreover, $E$ is locally asymptotically 
stable if $1<\beta<\beta_{c}$ and $E$ is a repeller if $\beta_{c}<\beta<\frac{1}{a}$. A NeimarkSacker bifurcation occurs at $\beta=\beta_{c}$ and the system has quasi-periodic solutions when $\beta>\beta_{c}$ and is close to $\beta_{c}$. In the numerical example given in Figure 2 for $a=0.5$ and $r=2.0$, we have $\beta_{c} \approx 1.3777$ and (2.2) has locally stable quasi-period solutions when $\beta \in\left(\beta_{c}, 1.42812\right)$. We expect that (2.2) undergoes a saddle node bifurcation when $\beta a=1$ so that the interior steady state $E$ disappears as $\beta$ is increased beyond $1 / a$.

- $\beta>1 / a$ : (2.2) has no interior steady state and $E_{0}$ is globally asymptotically stable if $\beta a>e^{\frac{1-a}{2}}$. Numerical simulations suggest that this global behavior remains true when $\beta a>1$.

Remark. From the above summary it is known that for a fixed $r$, $0<r<r_{0}$, there exists a unique $\beta_{c} \in(1,1 / a)$ such that $E$ is locally asymptotically stable if $\beta \in\left(1, \beta_{c}\right)$ and $E$ is a repeller when $\beta \in\left(\beta_{c}, 1 / a\right)$. A Neimark-Sacker bifurcation occurs at $\beta=\beta_{c}$ and the system has quasiperiodic solutions when $\beta>\beta_{c}$ and is close to $\beta_{c}$. As $r$ increases, the critical value $\beta_{c}$ also increases according to the numerical investigations and therefore the $\beta$ parameter region for which $E$ is locally asymptotically stable becomes larger. For instance, when $a=0.5$, we have $\beta_{c} \approx 1.318,1.3378$, $1.35765,1.3777,1.41819,1.45519,1.4589$ as $r$ increases from $0.5,1.0,1.5$, $2.0,3.0,3.9$ to 3.99 respectively. So if we fix $\beta=1.5$ and $r=3.99$, then since $\beta$ is close to 1.4589 , the system has a stable quasi-periodic solution as shown in Figure 3(a) with initial condition $(0.7512,0.2437)$ chosen to be close to the interior steady state. On the other hand, if $r$ is decreased to $r=0.5$, then since $\beta=1.5$ is somewhat much larger than the $\beta_{c}$ value of 1.318 , dynamics of $(2.2)$ behave much like Figure $2(\mathrm{~d})$. Solutions spiral away from the interior steady state and move toward $E_{0}$. We also plot the stable invariant loop solution in Figure 3(b) for $r=0.5, \beta=1.325$ and initial condition $(0.7811,0.0308)$.

\subsection{Existence and local stability of the interior steady states when $r>r_{0}$ and $\beta>1$}

In this subsection we study stability of interior steady states using Jury conditions [3]. Let $E=(x, y)$ be an interior steady state of (2.2). Recall that such an interior steady state exists if $a<1 / \beta<1$. The Jacobian matrix $J(E)$ evaluated at $E$ is given by (3.11) with trace and determinant given by (3.12) and (3.13) respectively. We separate the discussion into two 
cases: $a<x<\hat{x}$ and $\hat{x} \leq x<1$.

Assume first $\hat{x} \leq x<1$ and let $D(x)$ be the determinant of $J(E)$ given by (3.15). Then $D(\hat{x})=\frac{\hat{y}}{1-e^{-\hat{y}}}>1$ and $\lim _{x \rightarrow 1^{-}} D(x)=1-r(1-a)<$ $1-r_{0}(1-a)=-1$. By the same argument as in the case of $r<r_{0}$ and $\beta>1$, it can be verified that $D^{\prime}(x)<0$ for $x \in[\hat{x}, 1)$. Hence there exists a unique $\check{x}$ in $(\hat{x}, 1)$ such that $D(\check{x})=1$ and

$$
D(x)>1 \text { on }[\hat{x}, \check{x}) \text { and } D(x)<1 \text { on }(\check{x}, 1] .
$$

Moreover, since $x \geq \hat{x}, \operatorname{tr} J<1+\operatorname{det} J$ holds trivially.

Let

$$
T(x)=\operatorname{tr} J+1+\operatorname{det} J=(2+r x(1+a-2 x))\left(1+\frac{y}{e^{y}-1}\right)+y .
$$

Then $-1-\operatorname{det} J<\operatorname{tr} J$ is equivalent to $T(x)>0$. A direct differentiation yields

$T^{\prime}(x)=r(1+a-2 x) \cdot A-2 x\left(1+\frac{y}{e^{y}-1}\right)+r^{2} x(1+a-2 x)^{2} \cdot \frac{(1-y) e^{y}-1}{\left(e^{y}-1\right)^{2}}$,

where

$$
A=2+\frac{y}{e^{y}-1}+2 \cdot \frac{(1-y) e^{y}-1}{\left(e^{y}-1\right)^{2}} .
$$

Observe that $(1-y) e^{y}-1<0$ for $y>0$, and

$$
A>\frac{1}{\left(e^{y}-1\right)^{2}}\left[(2(1+y)-(y+2)) e^{y}-y\right]=\frac{y\left(e^{y}-1\right)}{\left(e^{y}-1\right)^{2}}>\text { for } y>0 .
$$

Therefore $T^{\prime}(x)<0$ for $x \in[\hat{x}, 1)$. Moreover, $T(\hat{x})=2\left(1+\frac{\hat{y}}{e^{\hat{y}}-1}\right)+\hat{y}>0$ and $\lim _{x \rightarrow 1^{-}} T(x)<0$ if $r>r_{0}$. Hence there exists a unique $\tilde{x} \in(\hat{x}, 1]$ such that

$$
T(x)>0 \text { on }[\hat{x}, \tilde{x}) \text { and } T(x)<0 \text { on }(\tilde{x}, 1] .
$$

In general, we assume $\check{x} \neq \tilde{x}$. Moreover, we do not consider the critical cases when either $x=\check{x}$ or $x=\tilde{x}$.

Theorem 3.8 Let $r>r_{0}, \beta>1$ and let $E=(x, y)$ be an interior steady state of (2.2) with $\hat{x} \leq x<1$. The following statements hold for $E$.

(a) If $\tilde{x}<\check{x}$, then (i) $E$ is a repeller if $\hat{x} \leq x<\tilde{x}$, (ii) $E$ is a saddle point if $\tilde{x}<x<\check{x}$, and (iii) $E$ is a saddle point if $x>\check{x}$. 
(b) If $\check{x}<\tilde{x}$, then (i) $E$ is a repeller if $\hat{x} \leq x<\check{x}$, (ii) $E$ is locally asymptotically stable if $\check{x}<x<\tilde{x}$, and (iii) $E$ is a saddle point if $x>\tilde{x}$.

Proof. (a) If $\hat{x} \leq x<\tilde{x}$, then $T(x)>0$ by (3.21) and $D(x)>1$ by (3.19). Therefore, $E$ is a repeller by a similar proof as that of Theorem 3.6. This proves (i). If $\tilde{x}<x<\check{x}$, then $T(x)<0$ and $D(x)>1$. Therefore, $\operatorname{tr} J<-2,(\operatorname{tr} J)^{2}-4 \operatorname{det} J>(1+\operatorname{det} J)^{2}-4 \operatorname{det} J=(1-\operatorname{det} J)^{2}>0$ and eigenvalues $\lambda_{ \pm}$of $J$ are real numbers. Since $T(x)<0$ and $\operatorname{det} J>1$, a direct computation shows $\lambda_{+}<1$ and $\lambda_{-}<-1$. Moreover, $\lambda_{+}>-1$ is satisfied since $-2-\operatorname{tr} J>0$ and $T(x)<0$. Therefore, $E$ is a saddle point, which proves (ii). Suppose now $x>\check{x}$. Then $D(x)<1$ and $T(x)<0$. If $-1-\operatorname{det} J \leq 0$, then $(\operatorname{tr} J)^{2}-4 \operatorname{det} J>0$. If $-1-\operatorname{det} J>0$, then $\operatorname{det} J<0$. Hence eigenvalues $\lambda_{ \pm}$of $J$ are real numbers. It is then straightforward to show that $E$ is a saddle point.

The proof of (b) is similar to (a). If inequalities in (i) are satisfied, then $D(x)>1, T(x)>0$ and thus $E$ is a repeller by an argument similar to the proof of Theorem 3.6. If $x \in(\check{x}, \tilde{x})$, then $D(x)<1$ and $T(x)>0$. Therefore $E$ is locally asymptotically stable by an earlier observation. If $x \in(\tilde{x}, 1)$, then $D(x)<1$ and $T(x)<0$. Therefore, $E$ is a saddle point by the proof of (a)(iii).

If the $x$ component of an interior steady state is such that $x<\hat{x}$, then

$$
\operatorname{det} J=(1+r x(1+a-2 x)) \beta x e^{-y}+y>\beta x e^{-y}+y=\frac{y}{1-e^{-y}}>1
$$

and such an interior steady state is always unstable.

Proposition 3.9 Let $r>r_{0}, \beta>1$ and let $E=(x, y)$ be an interior steady state of (2.2) with $x<\hat{x}$. Then $E$ is unstable.

However, since $r>r_{0}$, the function $\frac{1}{\beta}(x)$ defined in (3.10) may not be strictly increasing on $(a, 1)$ as in the case of $r<r_{0}$. Therefore, (2.2) may have multiple interior steady states. Furthermore, let

$$
Y(x)=g(x)-h^{-1}(x),
$$

where $g$ and $h$ are defined in (3.5) and (3.6) respectively. Then a tedious computation shows that

$$
Y^{\prime \prime \prime}(x)>0 \text { on }(0, \infty)
$$


Therefore, $Y(x)=0$ has at most three positive solutions. It follows that (2.2) has at most three interior steady states. There are plenty of parameter values for which there are two interior steady states. Although the analytical result indicates that the system can have at most three interior steady states, we do not find the existence of three interior steady states numerically. We conjecture that the system has at most two interior steady states when $r>r_{0}$ and $1<\beta<1 / a$.

Dynamics of (2.2) are complicated as illustrated by a numerical example with $a=0.1$ and $r=3.0>r_{0}$ given in Figure 3 . Notice that both populations become extinct when $\beta$ is large. It is suspected that the large turnover of parasitoids will drive the host population to below its Allee threshold so that the host population goes extinct and then the parasitoid population will inevitably become extinct.

The following table provides notation used in Sections 2 and 3 and Table 2 summarizes existence and stability of the interior steady states.

Table 1: Notation

\begin{tabular}{|c|l|}
\hline Notation & Definition \\
\hline$\hat{x}$ & $\frac{1+a}{2}$ \\
\hline$x_{m}$ & The critical point of $f(x)$ \\
\hline$x_{a}$ & The $x$ value for which $f(x)=a, x_{a}>\max \left\{x_{m}, 1\right\}$ \\
\hline$\underline{x}$ & The $x$ value for which $D(x)=1$ when $r<r_{0}$ and $\beta>1$ \\
\hline$\check{x}$ & The $x$ value for which $D(x)=1$ when $r>r_{0}$ and $\beta>1$ \\
\hline$\tilde{x}$ & The $x$ value for which $T(x)=0$ when $r>r_{0}$ and $\beta>1$ \\
\hline
\end{tabular}

Table 2: Existence and stability of an interior steady state $E=(x, y)$

\begin{tabular}{|c|l|l|}
\hline \multicolumn{1}{|c|}{$r$} & Existence & Stability \\
\hline$r<r_{0}$ & $a<\frac{1}{\beta}<1$ & $\begin{array}{l}\text { Locally asymptotically stable if } x>\underline{x} \\
\text { Repeller if } x<\underline{x}\end{array}$ \\
\hline$r>r_{0}$ & $a<\frac{1}{\beta}<1$ & $\begin{array}{l}\text { Locally asymptotically stable if } \check{x}<x<\tilde{x} \\
\text { Saddle point if } \tilde{x}<x<\check{x} \text { or } x>\check{x}>\tilde{x} \text { or } x>\tilde{x}>\check{x} \\
\text { Repeller if } \hat{x} \leq x<\tilde{x}<\check{x} \text { or } \hat{x} \leq x<\check{x}<\tilde{x}\end{array}$ \\
\hline
\end{tabular}




\section{Discussion}

Hosts and parasitoids are frequently insect populations with distinctive life stages and therefore discrete-time models are appropriate to describe such populations. Allee effects are biological phenomena which arise when reduced fitness or declined population growth occurs at low population densities or sizes. Mechanisms of Allee effects include failure of finding mates to reproduce or lack of cooperative individuals to explore resources collaboratively. When a population is subject to Allee effects, it is well known that there exists a critical population level below which the population will go extinct [5]. Consequently, studying population interactions involving Allee effects becomes an important subject of contemporary research.

In this investigation, we first study a single host population that follows the classical Ricker function and is also subject to Allee effects. The incorporation of Allee effects into the Ricker equation is different than those of the Ricker models with Allee effects in [9, 11, 12, 14, 17] but is similar to that given in [2, 16]. It is proved that there exists a threshold $r_{0}$ in terms of the Allee threshold $a$ such that the population either goes to extinction or stabilizes at the carrying capacity if its intrinsic growth rate $r$ is less than $r_{0}$. The basins of attraction of the local attractors are determined in Theorems 2.2 and 2.3. A period-doubling bifurcation occurs at $r=r_{0}$ (cf. Proposition 2.1), and, by numerical simulations, the host population will undergo a cascade of period-doubling bifurcations and eventually be chaotic if $r$ is increased further.

For the host-parasotoid system (2.2), it is shown in Proposition 3.1 that both populations will go extinct if the initial host population is either less than $a$ or is greater than the level of $x_{a}$. The only interesting dynamical behavior occurs when the initial host population is between $a$ and $x_{a}$. Moreover, the parasitoids will become extinct if $\beta$ is less than 1 (cf. Proposition 3.4). When $\beta$ exceeds 1 and $r<r_{0}$, then the interaction can support at most one interior steady state by Theorem 3.5. The interior steady state can be either locally asymptotically stable or is a repeller as illustrated in Theorem 3.6. If $r>r_{0}$, then the system may have multiple interior steady states. We provide linear stability analysis of these interior steady states in Theorem 3.8 and Proposition 3.9.

To compare the present investigation with other studies mentioned in the Introduction, we notice that [7] is on stochastic models while [6, 20, 21] deal with continuous-time models. Discrete-time models of Allee effects include [9, 10, 11, 12, 14, 17, 19, where [9, 14, 19] are of single population models and [12, 17] are of competition models. Moreover, the predator-prey model 
in [10] uses a Beverton-Holt growth equation. We therefore compare the present study with the model in [11]:

$$
\left\{\begin{array}{l}
N(t+1)=N(t) e^{r-N(t)} \frac{N(t)}{m+N(t)} e^{-b P(t)} \\
P(t+1)=\beta N(t)\left(1-e^{-b P(t)}\right),
\end{array}\right.
$$

where $1 / m>0$ is an individual host's searching efficiency. There exists a threshold $\tilde{r}_{0}$ as an increasing function of $m$, such that $E_{0}=(0,0)$ is globally asymptotically stable if $r<\tilde{r}_{0}$. The system has two more boundary states $E_{1 i}=\left(\bar{N}_{i}, 0\right), i=1,2$, when $r>\tilde{r}_{0}$, where $\bar{N}_{1}<\bar{N}_{2}$ depends on both $r$ and $m$. The host population goes extinct and so does the predator population if the initial host population is smaller than $\bar{N}_{1}$. System (4.1) may have multiple interior steady states and the system can either undergo a period-doubling bifurcation or a Neimark-Sacker bifurcation when an interior steady state loses its stability. Observe that global extinction also occurs in system (2.2) when $r<r_{0}$ and $\beta a>1$. The extinction of both populations in (2.2) is likely due to the predator's overexploitation as $\beta$ has to be large. Global asymptotic stability of $E_{0}$ in (4.1), however, is more likely to occur if an individual host's searching efficiency is small, i.e., if $m$ is large, so that $r<\tilde{r}_{0}$ is more likely to hold. Furthermore, (2.2) can only undergo a Neimark-Sacker bifurcation when $r<r_{0}$ while (4.1) can exhibit a period-doubling bifurcation (cf. Fig. 3(c)-(d) of [11]) when an interior steady state loses its stability.

Acknowledgements. We thank both referees for their valuable comments and suggestions. Y. Chow acknowledges the support of the National Science Council of Taiwan. S. Jang thanks the Institute of Mathematics, Academia Sinica, Taiwan for its financial and staff support for her winter 2012 and summer 2013 visits.

\section{References}

[1] Allee, W. C., The Social Life of Animals, William Heinemann, London, 1938.

[2] Allen, L., Fagan, J., Hognas, G., Fagerholm, H., Population extinction in discrete-time stochastic population models with an Allee effect, $J$. Difference Equ. Appl., 11, 273-293, 2005.

[3] Allen, L., An Introduction to Mathematical Biology, Prentice-Hall, New Jersey, 2006. 
[4] Amarasekare, P., Allee effects in metapopulation dynamics, Am. Nat., 152, 298-302, 1998.

[5] Courchamp, F., Berec, L., Gascoigne, J., Allee Effects in Ecology and Conservation, Oxford University Press, New York, 2008.

[6] Cushing, J.M., The Allee effect in age-structured population dynamics. In: T. Hallam, L. Gross and S. Levin (Eds.) Mathematical Ecology, 479-505, 1988. (New York: Springer-Verlag).

[7] Dennis, B., Allee effects in stochastic populations, Oikos, 96, 389-401, 2002.

[8] Elaydi, S., An Introduction to Difference Equations, 3rd edition, Springer, New York 2005.

[9] Elaydi, S., Sacker, R.J., Population models with Allee effect: a new model, J. Biol. Dyn., 4, 397-408, 2010.

[10] Jang, S. R.-J., Allee effects in a discrete-time host-parasitoid model, J. Diff. Equ. Appl., 12, 165-181, 2006.

[11] Jang, S. R.-J., Diamond, S., A host-parasitoid interaction with Allee effects on the host, Comput. Math. Appl., 53, 89-103, 2007.

[12] Kang, Y., Scramble competitions can rescue endangered species subject to strong Allee effects, Math. Biosci., 241, 75-87, 2013.

[13] Keitt, T., Lewis, M., Holt, R., Allee effects, invasion pinning, and species borders, Am. Nat., Vol. 157, 203-216, 2001.

[14] Li, J., Song, B., Wang, X., An extended discrete Ricker population model with Allee effects, J. Diff. Equ. Appl., 13, 309-321, 2007.

[15] Thieme, H.R., Dhirasakdanon, T., Han, Z., Trevino, R., Species decline and extinction: synergy of infectious diseases and Allee effect? J. Biol. Dyn., 3, 305-323, 2009.

[16] Liebhold, A., Bascompte, J., The Allee effect, stochastic dynamics and the eradication of alien species, Ecol. Lett., 6, 133-140, 2003.

[17] Livadiotis, G., Elaydi, S., General Allee effect in two-species population biology, J. Biol. Dyn., 6, 959-973, 2012. 
[18] Ricker, W.E., Stock and recruitment, J. Fish. Res. Board Can., 11, 559-623, 1954.

[19] Schreiber, S., Allee effects, extinctions, and chaotic transients in simple population models, Theor. Pop. Biol., 64, 201-209, 2003.

[20] Zhou, S. Wang, G., Allee-like effects in metapopulation dynamics, Math. Biosci., 189, 103-113, 2004.

[21] Zhou, S., Liu, Y., Wang, G., The stability of predator-prey systems subject to the Allee effects, Theo. Pop. Biol., 67, 23-31, 2005. 